\title{
COSMÉTICOS FACIAIS QUE CONTÊM PARABENO: QUAIS EFEITOS COLATERAIS PODEM CAUSAR NA PELE?
}

\section{ARTIGO DE REVISÃO}

NASCIMENTO, Suzana Maria do ${ }^{1}$

SALES, Gabriela Cecilia Paz ${ }^{2}$

SOUZA, Patrícia Gomes de ${ }^{3}$

LIMA, Patrícia Cristina Rodrigues ${ }^{4}$

NASCIMENTO, Suzana Maria do. Et al. Cosméticos faciais que contêm parabeno: Quais efeitos colaterais podem causar na pele? Revista Científica Multidisciplinar Núcleo do Conhecimento. Ano 05, Ed. 07, Vol. 03, pp. 83-97. Julho de 2020. ISSN: 2448-0959, Link de acesso: https://www.nucleodoconhecimento.com.br/saude/cosmeticos-faciais

\section{RESUMO}

Este artigo aborda a utilização de cosméticos faciais que contêm na formulação conservantes da classe dos parabenos. O proposito deste estudo é identificar os efeitos colaterais que estes conservantes podem ocasionar à saúde, ao serem aplicados sobre a pele. Este designo tem como base uma revisão bibliográfica, realizada através de livros e artigos científicos online. Nesta análise é possível compreender os conservantes mais comuns presentes nas formulações, bem como,

\footnotetext{
${ }^{1}$ Graduada em Estética e Cosmética UNIT.

${ }^{2}$ Graduada em Estética e Cosmética UNIT.

${ }^{3}$ Graduada em Estética e Cosmética UNIT.

4 Orientadora. Mestra em patologia UFPE. Especialista em Docência do Ensino Superior Fafire. Graduada em Estética e Cosmética UNIT.
} 
o limite de porcentagem, sua toxicidade e os efeitos colaterais acumulativos no organismo humano.

Palavras chaves: Parabenos, pele, microrganismo, conservantes.

\section{INTRODUÇÃO}

Atualmente existem vários produtos faciais disponíveis no mercado, com múltiplos benefícios. Ao serem aplicados sobre a pele, os cosméticos interagem com os queratinócitos presentes na epiderme, com os folículos pilosos e as glândulas sudoríparas. Este processo é chamado de permeação cutânea, no qual o ativo estabelece sua função desejada na pele. (LEONARDI, 2008).

Os primeiros povos a produzir e utilizar cosméticos foram os egípcios, há aproximadamente 30.000 anos (LEONARDI, 2008). Já em nosso país, as marcas AVON e L'Oréal chegaram só na década de 50. Nos anos 90, os primeiros produtos com ativo antienvelhecimento foram lançados (GALEMBECK; YARA, 2011). Atualmente os cosméticos são classificados de acordo com o grau de risco(sendo 1 ou 2), com intuito de proporcionar segurança ao consumidor (RIBEIRO, 2013).

Para conservar os produtos e mantê-los livres de microrganismos, bem como, prolongar sua boa qualidade, a indústria utiliza conservantes, em especial a classe dos parabenos (REBELLO, 2005). Os mais comuns entre as formulações são o metilparabeno e o propilparabeno e devem respeitar o limite de porcentagem que os conservantes devem ter ao serem adicionados nos cosméticos (SONI et al., 2002). Um produto ao ser aplicado pode causar irritabilidade na pele, isso é chamado de reação imunológica e as principais disfunções são as dermatites (SANTOS, 2008).

Este estudo analisa os conservantes da classe parabenos nas formulações cosmetológicas, visto que, muitas pessoas sofrem reações alérgicas ou desencadeiam patologias cutâneas como dermatites e acne ao utilizar cosméticos faciais com esses compostos. Além disso, o profissional da estética deve compreender as reações adversas que podem ocorrer na pele antes de adquirir 
produtos com estes ingredientes. O esteticista deve compreender toda fisiologia da pele e os diferentes tipos antes de aplicar qualquer ativo.

Apesar das reações que possam ser desencadeadas por tais ativos, é necessário que os produtos cosmetológimcos contenha conservantes, para inibir a proliferação de microrganismos e mantê-los seguros para uso por um determinado tempo. Desta forma, este artigo de revisão bibliográfica tem como objetivo analisar os efeitos colaterais que podem estar atrelados ao uso de cosméticos com parabenos e identificar uma forma segura deste composto químico ser adicionado à formulação, sem causar danos cutâneos.

\section{REFERENCIAL TEÓRICO}

\subsection{FISIOLOGIA TEGUMENTAR}

A pele é considerada o maior órgão do corpo humano, ocupando em média $2 \mathrm{~m}^{2}$, que corresponde cerca de $10 \%$ a $15 \%$ do peso total; esse órgão impede que microrganismos, produtos químicos, físicos ou alguns fatores ambientais como o sol, cheguem a entrar em contato danoso com o organismo. Outras funções vitais da pele são a termorregulação, que é encarregada de controlar a temperatura corporal, equilibrando a evaporação da água e a função metabólica, responsável pela síntese da vitamina D. Finalmente, a função sensorial, através da capitação de sensações pelos receptores sensoriais interligados ao sistema nervoso, corresponde ao tato. (LEONARDI, 2008).

A cútis é formada por 3 tipos de tecidos diferentes que trabalham em conjunto. $O$ tecido mais externo é a epiderme, que possui tecido epitelial pavimentoso e estratificado. Ela se subdivide em 5 camadas, sendo as da face respectivamente, córnea, granulosa, espinhosa e a basal ou germinativa (RABEH et al., 2013). Conforme Ribeiro (2010) na epiderme, além das células que dão forma ao tegumento, são encontradas também queratinócitos (que produzem queratina), melanócitos (produtores de melanina), células merkel (responsáveis pela sensação do tato) e langerhans (que possuem antígenos). 
O segundo tipo de tecido que compõe a pele é a derme, localizada medialmente logo abaixo da camada basal. A derme fornece resistência e elasticidade ao corpo contra traumas mecânicos. Dividida em derme papilar, que possui tecido conjuntivo frouxo e derme reticular constituído por tecido conjuntivo denso não modelado. Altamente vascularizada, essa camada fornece nutrientes para a epiderme, por possuir vasos linfáticos e sanguíneos, além de, fibroblastos, glândulas sebáceas e sudoríparas, folículo pilosos e terminações nervosas (LEONARDI, 2008). Encontra-se nessa região estruturas de fibras proteicas de colágeno e elastina, interligadas a uma matriz extracelular contendo glucosaminoglicanas, proteoglucanas e glucoproteínas (RIBEIRO, 2010).

A terceira e última camada é a hipoderme, uma tela subcutânea, constituída por tecido conjuntivo frouxo e pelas células adipócitarias. Sua função além de ser um deposito de reserva de energia, proporciona um isolamento térmico e proteção mecânica contra traumas, pressões e auxilia na mobilidade da pele em relação as camadas posteriores (LEONARDI, 2008).

\subsubsection{REAÇÕES IRRITATIVAS}

Atualmente há no mercado de cosméticos muita variedade de produtos e isso faz com o que os consumidores possam ter novidades e marcas diversas disponíveis. No entanto, muitas vezes, o usuário desconhece fatos imprescindíveis sobre as formulações e substâncias contidas nos cosméticos, correndo o risco de acarretar reações adversas com o uso das substâncias à venda. Harris (2005 apud CANDIOTTO, 2010) afirma que atualmente cerca de $20 \%$ da população apresenta algum tipo de dermatite, sendo a área da face a mais acometida e as mulheres a maioria das vítimas, através de produtos para o cuidado da pele e cabelo.

As alterações mais ocorridas no organismo são as alergias, que são nomeadas como reações imunológicas, adquiridas por certos tipos de substâncias contidas em alimentos ou cosméticos, que mesmo que em pouca quantidade possuem a capacidade de estimular algum tipo de irritação. É importante salientar que ao ser 
acometido por qualquer processo alergênico, deve-se suspender o uso do produto e procurar um profissional habilitado da área (SANTOS, 2008).

\subsubsection{ALERGIA E IMUNOLOGIA}

Quando o organismo sofre um processo alérgico, acontece conforme Galembeck; Csordas (2011) uma combinação de uma substância exógena com algumas proteínas do organismo, causando falhas bioquímicas ou enzimáticas, cuja intensidade depende da genética. Com isso, o cérebro começa a produzir histamina, por interpretar que a substância presente se trata de um invasor, buscando neutralizar essa tal agressão. Na maioria dos casos essa defesa causa uma diminuição da imunidade e elevação a propensão a doenças nos indivíduos.

As reações alérgicas mais comuns a partir dos cosméticos são as dermatites, sendo as principais:

- Dermatite de contato: esse tipo de irritação é a mais frequente. Geralmente ocorre um processo inflamatório não imunológico como resposta, sendo o tratamento delimitado à aplicação direta ao local do produto. Suas características são eritemas, vesiculações, prurido, pinicações que podem levar à destruição do tecido. "Estas reações surgem principalmente devido aos agentes irritantes que extraem os lipídios do extrato córneo e danificam até matar alguns queratinócitos" (BRASIL, 2010 apud CANDIOTTO, 2010, p.6).

- Dermatite alérgica: depois do contato com a substância alergênica há uma resposta imunológica. O sistema imune age contra a agressão, aumentando a produção de anticorpos, para que quando novamente for utilizado provoque uma reação inflamatória (HARRIS, 2005 apud CANDIOTTO, 2010). Pode haver manifestação de urticária como ação imediata ou hipersensibilidade se for a longo prazo. Por se tratar do mecanismo imunológico, essas afecções podem surgir em regiões diferentes da aplicação do produto (CHORILLI et al, 2007).

- Dermatite atópica: de acordo com Harris (2005, apud CANDIOTTO, 2010, p.8) "é uma enfermidade crônica, que ocorre em indivíduos que já possuem distúrbios dermatológicos". Por herança genética, por exemplo o indivíduo 
estará propenso a desenvolver este tipo de dermatite, tendo como características principias a coceira e prurido.

\subsection{COSMÉTICOS FACIAIS}

\subsubsection{HISTÓRIA E EVOLUÇÃO}

O uso de cosméticos data de aproximadamente 30.000 anos atrás, onde através dos relatos históricos, os egípcios se destacam como os primeiros a criarem produtos corporais e faciais. Para se protegerem da radiação solar eles utilizavam como base para os cremes a gordura animal e vegetal, cera de abelha e mel; Cleópatra se banhava em leite de cabra para obter uma pele macia e hidratada (LEONARDI, 2008).

Com o passar dos séculos os produtos foram aprimorados tendo o seu ápice no Brasil na década de 50, quando marcas multinacionais como AVON e L'Oréal foram trazidas para o país e começaram a comercializar produtos de beleza para todo o púbico. Nos anos 90 começam de fato a ser lançados os dermocosméticos, onde o tempo para o resultado foi reduzido consideravelmente, de um mês para 24 horas, resultando a partir do século 21 em diante no surgimento de cosméticos avançados, com alta tecnologia e multifuncionais, para o cuidado com a pele na estética. (GALEMBECK et al., 2011).

Por conta disso, é notório que a cosmetologia venha passando por várias evoluções a partir das ciências básicas e aplicadas em busca de inovações e resultados satisfatórios. Hoje há cosméticos com o poder de permeação dos ativos por vias transepidérmica, transcelular, intercelular, transfolicular e transglandular (LEONARDI, 2008).

Segundo a Agência Nacional de Vigilância Sanitária RDC № 211 (2005), um produto cosmético é qualquer substância ou mistura, designada a ter contato com as partes superficiais do corpo, como a epiderme, pelos e seus anexos, lábios, órgãos genitais externos, dentes e as mucosas bucais. Por isso, seu propósito é de ter a capacidade 
de higienizar, perfumar, melhorar o aspecto, proteção, equilibrar odores e mantê-las em boas condições.

Os produtos possuem classificações de acordo com o risco que oferecem ao consumidor:

- Grau 1 - risco mínimo, cosméticos, higiene pessoal, perfumes, com propriedades básicas, não havendo muitos detalhes de uso ou restrições.

- Grau 2 - risco potencial, destinados a indicações especificas com a exigência de comprovação e eficácia, modo, contraindicação e informações (RIBEIRO, 2013).

\subsubsection{NANOCOSMÉTICOS}

São estruturas que tem como base materiais em escala atômica. Entretanto, não é só a redução de escala que o difere dos demais cosméticos, mas também suas propriedades diferentes, devido ao tamanho menor do material. (SILVEIRA et al., 2018). Tem como proposta; melhorar o desempenho dos cosméticos proporcionando melhor permeabilidade cutânea.

Por conter pequenas partículas, autores descrevem que produtos com nanotecnologia, carregam ativos para a derme, estabilizam e protegem os ativos contra contaminação e degradação e, além disso, visam minimizar os ricos de irritação na pele. (ZYCHAR; KATAOKA; AUDI, 2016 apud SILVEIRA, 2018).

Alguns autores pontuam que estes ativos com nanopartículas podem gerar efeitos cumulativos no organismo, como estresse oxidativo no sistema nervoso humano, por penetrar em camadas mais profundas. Um número maior de pesquisas sobre o assunto faz-se necessário para que haja clareza nas conclusões do que podem de fato ocasionar no organismo a longo prazo o uso desses ativos. (SRIVASTAVA; GUSAIN e SHARNA 2015 apud SILVEIRA, 2018). 


\subsubsection{CONSERVANTES MICROBIANOS}

Conforme o código da ANVISA RDC № 162 (2001) descreve, conservantes são substâncias adicionadas como ingrediente aos produtos de higiene pessoal, cosméticos e perfumes com a finalidade de inibir o crescimento de microrganismos durante sua fabricação e estocagem, assegurando dessa forma a validade do produto e evitando contaminações dos mesmos.

Um produto infectado por micróbios, apresenta uma modificação na coloração, odores e em alguns casos mais graves mudanças na viscosidade, desestabilização física e química do cosmético. A busca de conservantes ideias para as formulações é algo que exige muita atenção, pois é necessário atender a todos os requisitos de segurança, toxidade, possuir um ph, temperatura e quantidades adequadas, sem alterar as características do produto ou ser agressivo ao consumidor (WILKINSON, et al., 1982 apud RIBEIRO, 2013).

\subsubsection{PARABENOS}

Os parabenos são substâncias com ação conservante, utilizados em cosméticos, perfumes, enlatados e produtos de higiene pessoal. São conservantes muito comumente utilizados, por inibir o crescimento de microrganismos e proteger os produtos contra oxidações indesejáveis. Os parabenos interagem com outras substâncias da fórmula, possuem uma alta estabilidade química, são biodegradáveis, possuem baixo custo e são de fácil sintetização, mantendo a qualidade do cosmético e segurança do uso (REBELLO, 2005).

Os parabenos mais utilizados em cosméticos são metilparabeno, propilparabeno, álcool benzílico e fenoxietanol. As regulamentações da União Europeia e do Brasil permitem o uso de no máximo $0,4 \%$ de cada parabeno e um máximo de $0,8 \%$ de parabeno total no cosmético. A atividade antimicrobiana desses compostos aumenta com o aumento da cadeia carbônica do substituinte do éster, mas sua solubilidade em água decresce proporcionalmente (SONI et al., 2002). 


\subsection{PRINCIPAIS PARABENOS}

\subsubsection{PROPILPARABENO - NIPAZOL}

Derivados do ácido p-hidroxibenzóico são largamente utilizados nas indústrias cosméticas e farmacêuticas, sendo juntamente com o nipagin, o conservante cosmético de maior aceitação. Possui baixa toxicidade, compatibilidade com uma gama de ingredientes e atividade em ampla faixa de ph que é usado na maioria das categorias de formulações cosméticas (SONI et al., 2002). Apresenta baixa solubilidade em água e solúvel na maioria dos óleos, emulsionantes, álcoois graxos e ceras, (CLARIANT, 2003).

\subsubsection{METILPARABENO - NIPAGIM}

Substância química, com concentrações de benzeno, hidroxila, metila e grupo éster, é utilizado como conservante e germicida, usado em cosméticos por ter ação eficaz contra bactérias, fungos e leveduras. Tem capacidade de solubilidade junto ao etanol, cetona e propilenoglicol. Entretanto, a solubilidade de metilparabeno em água é obtida com mais dificuldade. Empregado em cremes, shampoos, loções, pastas, géis, desodorantes, maquiagem para a área dos olhos, condicionadores, produtos infantis, cremes dentais, entre outros. (CLARIANT, 2010)

\subsubsection{FENOXIETANOL}

Apresenta uma grande eficácia na ação contra microorganismos, é facilmente incorporado nas formulações pois é solúvel em diversos ingredientes lipossolúveis, tem boa solubilidade em água, solúvel em álcool, éter, acetona, glicerol, propilenoglicol, soluções de hidróxido de sódio, e pouco solúvel em óleos minerais. (CLARIANT, 2003) 


\subsection{4 ÁLCOOL BENZÍLICO}

O álcool benzílico ou fenilmetanol é um conservante que possui atributos letais para microrganismos pela sua competência de modificar as membranas e coibir a síntese de proteínas (LUCHINNI et al., 1990). Também é manuseado como ingrediente de aromas e fragrâncias. (SCOGNAMIGLIOA et al., 2012).

\section{MATERIAIS, MÉTODOS E DISCUSSÃO}

A presente pesquisa foi executada através de uma revisão bibliográfica. Foram utilizadas como fonte de pesquisa a plataforma Scielo, revistas brasileiras dentro das áreas da iniciação cientifica, otorrinolaringologia e ciência farmacêutica e bases de pesquisa internacional como Clarieant, NCBI (National Center for Biotechnology Information) e ScienceDirect.

Ademais, foram empregadas pesquisas com base em livros, websites e artigos científicos publicados nos bancos de pesquisa das universidades brasileiras. Foram analisados conteúdos nacionais e internacionais, publicados entre o períodos de 1999 a 2018. As pesquisas por artigo científico tiveram como palavras-chave: parabeno, conservantes, pele e microrganismo.

Desse modo, dos 30 artigos analisados, apenas 16 estavam relacionados ao tema e 14 foram excluídos. Desses 14 artigos eliminados, 10 ficaram de fora por não conter informações relacionado ao tema, 3 por não ter origem de pesquisa confiável e 1 por ser duplicado. Durante o estudo foi notória a escassez de conteúdo relevante sobre o assunto, tornando a elaboração da presente pesquisa ainda mais desafiadora.

\subsection{TOXICIDADE DOS PARABENOS E SEUS EFEITOS CUMULATIVOS NO ORGANISMO}

Os parabenos são exemplos de substâncias que podem acarretar efeitos indesejáveis no organismo por promoverem sensibilização e processos alérgicos na pele como dermatites de contato, promotores da adipogênese, desregularização endócrinas, 
exposição ao feto e há até alguns relatos de câncer de mama, podendo ainda ter ação semelhante à do estrogênio. Porém, ainda há muita controvérsia em relação aos parabenos. (HARRIS, 2005 apud CANDIOTTO, 2010).

Segundo estudo de Edja Querino e Roberta Silva (2018) quando os parabenos são metabolizados, estes se convertem parte em ácido p-hidroxibenzóico, cuja estrutura está relacionada ao ácido acetilsalicílico. Essa substância pode causar reações de hipersensibilidade e em muitos casos envolve reações de contato em formulações cosméticas em que haja a combinação de metilparabeno com proprilparabeno. Essas irritações podem aparecer tanto no local de aplicação do produto, como em outros locais do corpo.

Rabello (2005) afirma que os parabenos são conservantes mais utilizados em cosméticos e produtos de higiene pessoal por possuir estabilidade Química, mantendo a qualidade do produto, além de possuir baixo custo. Entretanto, Harris (2005) alega que o uso contínuo de cosméticos com parabeno na formulação leva ao acúmulo dessa substância na derme, resultando em dermatite de contato, causada pela resposta do sistema imune do organismo contra agressão do produto aplicado. Com isso, o primeiro caso de alergia pelo parabenos, segundo a literatura, surgiu no ano de 1940, causado pelo etilparabeno contido no creme fungicida, ou seja, mesmo que a exposições humana a essa substância aconteça por diversos tipos de produtos, o principal meio de contato são os cosméticos faciais e capilares e as alergias e dermatites de contato são as principais complicações, acometendo mais a região da face. (QUERINO; SILVA, 2018 apud MOWARD, 2000).

Chorilli (2007) declara que a dermatite alérgica pode surgir ao longo do tempo e até mesmo em áreas do corpo que não receberam a aplicação do cosmético, por consequência do mecanismo imunológico do organismo. Edja Querino e Roberta Silva (2018) destacam que a combinação do metilparabeno com proprilparabeno pode causar reações irritativas, surgindo não só no local da utilização do produto, mas em outras regiões do corpo. Porém, Ribeiro (2013) admite que é de suma importância a adição de componentes nas formulações que conservem o cosmético por mais tempo, 
pois um produto infectado por microrganismos devido a falta de conservantes apresenta mudança na viscosidade, odores e coloração.

É notório que a ANVISA permita no máximo $0,8 \%$ de quantidade total de parabeno por produto. No entanto, o perigo está na fração de vezes em que os cosméticos são aplicados diariamente, desse modo, multiplicando o volume de concentração dos parabenos depositados na pele, que possuem o efeito acumulativo na derme (SONI et al., 2002). Por isso, o intuito desse artigo é alertar o consumidor sobre a diminuição da quantidade de produtos com este tipo de conservante na formulação, para que ao utilizar no cotidiano, a soma desses produtos não ultrapasse o limite de parabenos determinada pela regulamentação.

\section{CONCLUSÃO}

Como foi dito anteriormente, os parabenos são compostos utilizados para conservação e inibição de microrganismos, principalmente em cosméticos. Com os estudos concluímos que os parabenos causam reações adversas com uso contínuo, como alergias, dermatites, alterações endócrinas e até câncer de mama, já que tem a predisposição de acumular-se nos tecidos (derme). Ou seja, concluímos que, o uso desse composto é nocivo a saúde e que deve ser substituído por outro componente que tenha a mesma aplicabilidade ou mesmo administrado em doses menores, que não cause danos à saúde e que diminua o potencial alergênico e irritante dos produtos.

\section{REFERÊNCIAS}

BALBANI, A. P. S.; STELZER, L. B.; MONTOVANI, J. C. Excipientes de medicamentos e as informações da bula. Revista Brasileira de Otorrinolaringologia, São Paulo, v.72, n. 3, 2006.

BRASIL, Ministério Da Saúde. Agência Nacional de Vigilância Sanitária: Guia de Estabilidade de Produtos Cosméticos, maio de 2004. Disponível em: <http: http://portal.anvisa.gov.br/documents/106351/107910/Guia+de+Estabilidade+de+Pro 
dutos+Cosm\%C3\%A9ticos/49cdf34c-b697-4af3-8647-dcb600f753e2>. Acessado em 29 de out. 2019.

BRASIL, Ministério Da Saúde. Agência Nacional de Vigilância Sanitária. Resolução RDC no 332 de 01 de dezembro de 2005. As empresas fabricantes e/ou importadoras de Produtos De Higiene Pessoal Cosméticos e Perfumes, instaladas no território nacional deverão implementar um Sistema de Cosmetovigilância, a partir de 31 de dezembro de 2005. Disponível em <http://www.anvisa.gov.br/e-legis>. Acessado em 25 de novem. 2019.

BRASIL. RDC n. 481, de 23 de setembro de 1999. Estabelece os parâmetros de controle microbiológico para os produtos de higiene pessoal, cosméticos e perfumes conforme anexo dessa Resolução. Diário Oficial da União. Brasília, DF, 27 set. 1999. Disponível em: <http://portal.anvisa.gov.br>. Acessado em 29 de out. 2019.

CHORILLI, M; SCARPA. M. V; CORRÊIA, M. A. Reações adversas a cosméticos infarma. São Paulo, v. 19, 2007.

CLARIANT. NipaginTM M. Conservante para a indústria cosmética. Functional Chemicals Division. 2010. Disponível em: < https://www.clariant.com/pt/BusinessUnits/Industrial-and-Consumer-Specialties/Clariant-Essentials/Mercados-deatuacao/Personal-Care/Criacao-de-Valor/Nipaguard >. Acessado em 17 de mar. 2020. Relatório técnico.

CLARIANT. Nipasol® M. Conservante para a indústria cosmética. Functional Chemicals Division. 2003. Disponível em: < https://www.clariant.com/pt/BusinessUnits/Industrial-and-Consumer-Specialties/Clariant-Essentials/Mercados-deatuacao/Personal-Care/Criacao-de-Valor/Nipaguard >. .Acessado em 17 de mar. 2020. Relatório técnico.

CLARIANT. Phenoxetol®. Conservante para a indústria cosmética. Functional Chemicals Division. 2003. Disponível em: < https://www.clariant.com/pt/BusinessUnits/Industrial-and-Consumer-Specialties/Clariant-Essentials/Mercados-de- 
atuacao/Personal-Care/Criacao-de-Valor/Nipaguard >. Acessado em 17 de mar. 2020. Relatório técnico

GALEMBECK, Fernando; CSORDAS, Yara. Cosméticos: a química da beleza. Disponível em: <http://old.agracadaquimica.com.br/quimica/arealegal/outros/175.pdf> Acessado em 29 de out. 2019.

HARRIS, M. I. Pele: estrutura, propriedades e envelhecimento. 2. ed. Rev. e ampl. São Paulo: SENAC, 2005 apud CANDIOTTO, A. A.; WAYHS, A. F.; FRANÇA, A. J. Reações adversas a cosméticos e o profissional da estética. p.4, 2010.

LEONARDI, Gislaine. Cosmetologia aplicada. 2.ed. São Paulo, 2008.

LUCCHINI, J. J.; CORRE, J.; CREMIEUX, A. Antibacterial activity of phenolic compounds and aromatic alcohols. Research in Microbiology. v. 141, p. 499-510, 1990. Disponível em: <http://www.sciencedirect.com/science/article/ pii/0923250890900752>. Acessado em 17 de mar. 2020.

MOWAD, C. Allergic contact dermatites caused by parabéns: 2 case reports and a review. American Journal of Contact Dermatitis, V. 11, 2000 apud QUERINO, Edja; SILVA, Roberta. Análise dos riscos à saúde, dos parabenos em cosméticos. Rio grande do norte, p.5, 2018.

QUERINO, Edja; SILVA, Roberta. Análise dos riscos à saúde, dos parabenos em cosméticos. Rio grande do norte, 2018.

RABEH, Soraia; GONÇALVES, Márcia. Material complementar: anatomia e fisiologia da pele. 2013.2 Disponível em: <https://edisciplinas.usp.br/pluginfile.php/2124718/mod_folder/content/0/1.\%20Anato mia\%20e\%20Fisiologia\%20da\%20Pele.pdf?forcedownload=1>. Acessado em 29 de out. 2019.

REBELLO, T. Guia de produtos cosméticos. 6ª edição. São Paulo: Senac, p.161, 2005. 
RIBEIRO, Brenda. Otimização de sistemas conservantes em bases cosméticas emulsionadas. Curitiba, 2013.

RIBEIRO, Cláudio. Cosmetologia aplicada a Dermoestética. 2.ed. São Paulo, 2010. SANTOS, Hamilton. Toxicologia: a garantia de cosméticos seguros. São Paulo, v. 20, n. 2, p.20-23, 2008.

SCOGNAMIGLIOA, J.; JONES, L.; VITALEA, D.; LETIZIAA, C.S.; API, A.M. Fragrance material review on benzyl alcohol. Food and Chemical Toxicology. p. $140-160$, 2012.

SILVA, A. V. A.; FONSECA, S. G. C.; ARRAIS, P. S. D; FRANCELINO, E. V. Presença de Excipiente com Potencial para Indução de Reações Adversas em Medicamentos Comercializados no Brasil. Revista Brasileira de Ciências Farmacêuticas, Ceará, v.44, n.3, 2008.

SILVEIRA, Camila. et al. ESTUdO CIENCIOMÉTRICO DE NANOCOSMÉTICO. 2018 . < https://periodicos.itp.ifsp.edu.br/index.php/IC/article/view/1116/1050> acessado em 27 de nov. 2019.

SOCIEDADE BRASILEIRA DE DERMATOLOGISTAS. Tipos de pele. 2017. Disponível em: <https://www.sbd.org.br/dermatologia/pele/cuidados/tipos-de-pele/>. Acessado em 02 out. 2019.

SONI, M. G.; TAYLOR, S. L.; GREENBERG, N. A.; BURDOCK, G. A. Evaluation of the health aspects of methylparaben: a review of the published literature. Food and Chemical Toxicology. v. 40, p. 1335-1373, 2002.

SRIVASTAVA, V.; GUSAIN, D.; SHARMA, Y. C. Industrial \& Engineering Chemistry Research. v. 54 n. 24, p.6209-6233, 2015 Apud SILVEIRA, C.; CORREIA, A.; SAMPAIO, L.; CARNEIRO, F. ESTUDO CIENCIOMÉTRICO DE NANOCOSMÉTICOS. $2018 . \quad$ Disponível em< 
https://periodicos.itp.ifsp.edu.br/index.php/IC/article/view/1116>. Acessado em 27 de nov. 2019.

TULLER, Gabriela. Tipos de pele. 2017. Disponível em: $<$ https://mail.google.com/mail/u/0/\#search/ebook/FMfcgxwChJjptSRWTNMmFsxPrPp XTwhV?projector=1\&messagePartld=0.2> Acessado em 02 de out. 2019.

WILKINSON, J.B.; MOORE, R.J. Harry's Cosmeticology, 7.ed. Nova lorque: Chemical Publishing, 1982 apud RIBEIRO, Brenda. Otimização de sistemas conservantes em bases cosméticas emulsionadas. Curitiba, 2013.

ZYCHAR, B. C.; KATAOKA, V. Y.; AUDI, C. A prospecção da nanotecnologia cosmética no setor da estética e suas principais nanoestruturas. v. 4, n. 4, p. 1 19, 2016 Apud SILVEIRA, C. et al. Estudo Cienciométrico De Nanocosmético. 2018. https://periodicos.itp.ifsp.edu.br/index.php/IC/article/view/1116> acessado em 27 de nov. 2019.

Enviado: Fevereiro, 2020.

Aprovado: Julho, 2020. 